\title{
Quartic monoid surfaces with maximum number of lines
}

\author{
Mauro Carlo Beltrametti ${ }^{1}$ \\ Dipartimento di Matematica, Università di Genova, Via Dodecaneso, 35, 16146 \\ Genova, Italy
}

Alessandro Logar ${ }^{2}$

Dipartimento di Matematica e Geoscienze, Università degli Studi di Trieste, Via Valerio 12/1, 34127 Trieste, Italy.

Maria Laura Torrente

Dipartimento di Economia, Università di Genova, Via Vivaldi 5, 16146 Genova, Italy

\begin{abstract}
In 1884 the German mathematician Karl Rohn published a substantial paper on [11] on the properties of quartic surfaces with triple points, proving (among many other things) that the maximum number of lines contained in a quartic monoid surface is 31 .

In this paper we study in details this class of surfaces. We prove that there exists an open subset $A \subseteq \mathbb{P}_{K}^{1}$ ( $K$ is a characteristic zero field) that parametrizes (up to a projectivity) all the quartic monoid surfaces with 31 lines; then we study the action of PGL $(4, K)$ on these surfaces, we show that the stabiliser of each of them is a group isomorphic to $S_{3}$ except for one surface of the family, whose stabiliser is a group isomorphic to $S_{3} \times C_{3}$. Finally we show that the $j$-invariant allows one to decide, also in this situation, when two elements of $A$ give the same surface up to a projectivity.

To get our results, several computational tools, available in computer algebra systems, are used.
\end{abstract}




\section{Introduction}

Algebraic quartic surfaces are a classical subject of algebraic geometry and the study of their rich properties has been developed in research papers and books since the XIX century. Many different classifications for several classes of quartic surfaces where introduced (for instance, see [11], the books [6] and [4]; for a more complete discussion, see [7, [10] and the references given there).

In more recent years, many classical results have been reconsidered and presented in a modern language. In particular, a big effort has been dedicated to study the possible singularities on quartic surfaces [5], the number of lines contained in quartic surfaces [7, and to study as well the characteristics of monoid surfaces, as in [13], [14, where an explicit description of them in terms of equations can be found (see also [9] and [2]). Unlike the well-known case of smooth cubic surfaces, which contain 27 lines, the generic quartic surface does not contain lines. However there are classes of quartic surfaces which do contain lines, and it has been shown that the maximum number is 64 (see the paper [7] and its references).

An interesting class of quartic surfaces is given by the quartic monoid surfaces, that is, surfaces with a triple point. Their classification in terms of other singularities is given in [13] and [14], while in the forthcoming paper [3], it is described a classification according to the possible configuration of lines they can contain. In particular, it is shown that a quartic monoid surface can contain at most 31 lines; indeed such a result was already obtained by Rohn in a paper published in 1884 (see [1]).

In this paper we aim to study into details the class of quartic monoid surfaces with 31 lines. In particular, we want to describe the natural action of the group $\operatorname{PGL}(4, K)$ on these surfaces, which means that we are interested in quartic monoid surfaces up to projectivity. We show that quartic monoid surfaces with 31 lines can be parametrized by an open subset $A$ of $\mathbb{P}_{K}^{1}$ and, moreover, that for any given points $a, b \in A$ the corresponing surfaces $Q(a)$ and $Q(b)$ are projectively equivalent if and only if $a$ and $b$ have the same $j$-invariant. Furthermore, if $a \in A$ is not a primitive root of -1 , then the stabiliser of the corresponding surface $Q(a)$ is a group isomorphic to the group of permutations $S_{3}$ of three elements (which does not depend on the parameter $a$ ), while if $a$

Email addresses: beltrame@dima.unige.it (Mauro Carlo Beltrametti), logar@units.it (Alessandro Logar), marialaura.torrente@economia.unige.it (Maria Laura Torrente).

1 Partially supported by project G.N.S.A.G.A. 2016, 2017 Geometria algebrica e algebra commutativa

2 Partially supported by the FRA 2018 grant "Aspetti geometrici, topologici e computazionali delle varietà", Università di Trieste 
is a primitive root of -1 , then the stabiliser of the corresponding surface is a group with 18 elements, isomorphic to $S_{3} \times C_{3}$, where $C_{3}$ denotes the cyclic group of order 3 . If the parameter $a$ is rational, then all the 31 lines of $Q(a)$ are rational (i.e. obtained by joining two points with rational coordinates). The approach we have followed is quite constructive so that we get (or we can easily obtain) the explicit equations of all the considered geometric objects. We have therefore intensively used symbolic computation tools, and precisely the computer algebra systems CoCoA [1] and Sage [12].

\section{Basic properties}

Let $K$ be a characteristic zero field. By a quartic monoid of $\mathbb{P}_{K}^{3}$ we mean a quartic surface of $\mathbb{P}_{K}^{3}$ which has a triple point (that, w.l.o.g. can be assumed the origin $O=(0,0,0,1))$. Hence the polynomial defining a quartic monoid is of the form:

$$
Q(x, y, z, t)=t F_{3}(x, y, z)+F_{4}(x, y, z)
$$

where $F_{3}, F_{4} \in K[x, y, z]$ are two forms of degree three and four, respectively. From now on, for shortness, we also denote by $Q$ the quartic surface of equation $Q(x, y, z, t)=0$.

Let $\pi$ be the plane of $\mathbb{P}_{K}^{3}$ of equation $t=0$. We consider on this plane the two curves of equation $F_{3}=0$ and $F_{4}=0$, which intersect in 12 points $P_{0}, \ldots, P_{11}$ on $\pi$. The quartic monoid $Q$ contains the 12 lines given by $O+P_{i}$. Moreover, if a line $r$ is contained in the surface $Q$ and does not pass through the origin, then its projection from $O$ to the plane $\pi$ gives a line joining three of the points $P_{i}$ 's, see [7] and [11] (indeed, the intersection $\{r+O\} \cap Q$ is a quartic planar curve which splits into $r$ and three other lines through the origin, which represent the three possible collinearities of three points of $\pi$ ). This remark has an immediate consequence: the number of lines a quartic monoid can contain depends on the collinearities of 12 points of the plane. In particular, Rohn claimed in [11] that the maximum number of collinear triplets of points among 12 points in the plane is 19, so that the maximum number of lines contained in a quartic monoid is 31 (19 from the collinearities, 12 from the lines through the singular point). He also gave an explicit (very elegant) equation of a quartic surface with 31 lines, which can be expressed (according to the formulation of [7]) in the form:

$$
t\left((x+y+z)^{3}+x y z\right)+(x+y+z)(x-y)(y-z)(z-x)
$$

In the present paper, we want to study into details the quartic monoid surfaces with maximum number of lines, with particular effort to describe their possible symmetries. 


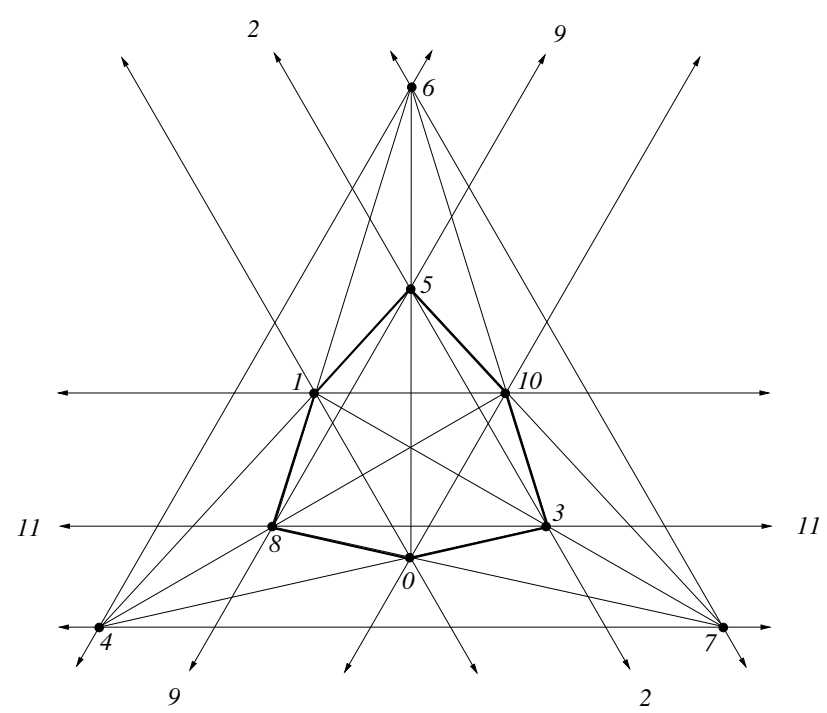

Fig. 1. The 19 collinearities of the points described by (3). They can be realized starting from the 15 diagonals of an hexagon (of vertices $0,3,10,5,1,8$ ) that meet in the further points 4,6,7 and 2,9,11 (these last points lie on the line at infinity).

First of all, the combinatorial problem of finding the maximum number of collinear triplets of 12 points of the plane can easily be solved with the help of a computer. The solution we get (unique, up to permutations of the labels) is the following (see also Figure 1) (where, from now on, $(i, j, k)$ is a shortcut to denote the triplet $\left.\left(P_{i}, P_{j}, P_{k}\right)\right)$ :

$$
\begin{aligned}
& (0,1,2),(0,3,4),(0,5,6),(0,7,8),(0,9,10),(1,3,7),(1,4,5), \\
& (1,6,8),(1,10,11),(2,3,5),(2,6,7),(2,9,11),(3,6,10), \\
& (3,8,11),(4,6,9),(4,7,11),(4,8,10),(5,7,10),(5,8,9)
\end{aligned}
$$

In order to make computations, it is necessary to assign coordinates to the points. Up to a projectivity of the plane $\pi$, we can assume that the coordinates $(x, y, z, t)$ of the points $P_{0}, P_{1}, P_{2}, P_{3}, P_{4}$ are the following:

$$
P_{0}, P_{1}, P_{2}, P_{3}, P_{4}=(0,0,1,0),(1,0,1,0),(2,0,1,0),(0,1,1,0),(0,2,1,0)
$$

The remaining points have to satisfy two constraints: they must satisfy conditions (3) and they have to lie on a plane cubic curve. Consequently, the only possible coordinates of the remaining points turn out to be expressed in terms of a free parameter $a \in K$ as follows:

$$
\begin{aligned}
& P_{5}, P_{6}, P_{7}=(1,1,3 / 2,0),(1,1,1 / 2 a+2,0),(a+1,1, a+2,0), \\
& P_{8}, P_{9}=(a+1,1,3 / 2 a+2,0),(1,-a+1,2,0), \\
& P_{10}, P_{11}=(1,-a+1,-1 / 2 a+2,0),(a+1,-a+1,1 / 2 a+2,0)
\end{aligned}
$$


An easy computation shows the following fact.

Lemma 1. The points $P_{0}, \ldots, P_{11}$ are all distinct if and only if $a \neq-1$ or $a \neq 0$. If the 12 points are distinct, then they satisfy the collinearities conditions given by (3) and no more if and only if $a \neq 1 / 2, a \neq 1$ or $a \neq 2$.

We denote by $D$ the set $\{-1,0,1 / 2,1,2\} \subseteq K$ corresponding to the degenerate cases of the points.

For a given $a \in K \backslash D$, the cubic curve $C_{3}(a)$ passing through the 12 points $P_{0}, \ldots, P_{11}$ has equation $F_{3}(a)=0$, where:

$$
\begin{aligned}
F_{3}(a)= & (a-1) x^{3}+\left(2 a^{2}+6 a-1\right) x^{2} y-3(a-1) x^{2} z+\left(a^{2}+4 a+1\right) x y^{2} \\
& +2(a-1) x z^{2}-2 a(a+4) x y z+(a+1) y^{3}-3(a+1) y^{2} z+2(a+1) y z^{2}
\end{aligned}
$$

In order to construct the quartic monoids, we need quartic curves of the plane $\pi$ passing through the 12 points. For a fixed $a \in K \backslash D$, let $\mathcal{L}_{a}$ be the linear system of quartic curves through the 12 points.

Lemma 2. The linear system $\mathcal{L}_{a}$ of quartic curves through the 12 points $P_{0}, \ldots, P_{11}$ has dimension 4 . If $\ell_{1}, \ell_{2}, \ell_{3}$ are three fixed, generic lines in the plane and if $r_{1}, r_{2}, r_{3}, r_{4}$ are the four lines passing through the triplets of collinear points $\left(P_{0}, P_{1}, P_{2}\right),\left(P_{3}, P_{6}, P_{10}\right),\left(P_{4}, P_{7}, P_{11}\right),\left(P_{5}, P_{8}, P_{9}\right)$, then a basis for the linear system $\mathcal{L}_{a}$ is given by the four quartics:

$$
C_{3}(a)+\ell_{1}, \quad C_{3}(a)+\ell_{2}, \quad C_{3}(a)+\ell_{3}, \quad r_{1}+r_{2}+r_{3}+r_{4}
$$

Proof. To compute the dimension of the linear system, it is enough to take a generic quartic curve of $\mathbb{P}^{3}$ and to impose that it passes through the 12 points. The computation of the rank of the $12 \times 15$ matrix associated to the system, gives the dimension of $\mathcal{L}_{a}$. In order to speed up the computation, it is convenient to observe that the points $P_{0}, \ldots, P_{5}$ have fixed coordinates. Therefore, to find the dimension of $\mathcal{L}_{a}$ we can solve six of the equations in terms of the others, hence it suffices to find the rank of a $6 \times 9$ matrix (whose entries are polynomials in $a$ ). It turns out that all the maximal minors are zero, while the $5 \times 5$ minors are zero if and only if $a=0$, which is excluded by our hypothesis. Finally, all the four quartic curves of (5), contain the 12 points and they are clearly linearly independent.

The equation of the quartic curve which splits into the lines $r_{1}, \ldots, r_{4}$ is:

$$
\begin{aligned}
F_{4}(a)= & y(3 a x-2 a z+x-y)(2 a x+a y-2 a z+3 x+y-2 z) \\
& (a x+2 x+2 y-2 z)
\end{aligned}
$$


Thus, as a consequence of the previous lemma one sees that all the quartic monoids with 31 lines (up to a change of coordinates) have equation:

$$
t F_{3}(a)+\left(\alpha_{0} x+\alpha_{1} y+\alpha_{2} z\right) F_{3}(a)+b F_{4}(a)=0
$$

where $a, \alpha_{0}, \alpha_{1}, \alpha_{2}, b$ are parameters in $K$. If we change the coordinates writing $t-\alpha_{0} x-\alpha_{1} y-\alpha_{2} z$ in place of $t$, we finally get that, up to a change of coordinates, all the quartic monoids with 31 lines have equation $Q(a, b)=0$, where:

$$
Q(a, b)=t F_{3}(a)+b F_{4}(a)
$$

with $a \in K \backslash D, b \neq 0$. Moreover, starting from the same given points $P_{0}, \ldots, P_{11}$, different values of $b$ give different quartic monoids.

We conclude this section with the following proposition, which shows that the surface (6) does not have singular points (apart from the origin).

Proposition 3. If $a \notin D$ and $b \neq 0$, then the only singular point of the quartic surface (6) is the origin.

Proof. If $T$ is a singular point of $Q=Q(a, b)$ different from the origin $O$, then the line joining $O$ and $T$ is contained in $Q$, therefore every singular point of $Q$ lies on one of the 12 lines of $Q$ passing through the origin. Hence we have to look for singular points among the points $T_{\lambda}^{(i)}=O+\lambda P_{i}$ (where $\lambda$ is a parameter). If we substitute $T_{\lambda}^{(i)}$ into the gradient of $Q$ and we saturate the ideal w.r.t. the polynomial $a(a-1)(a+1)(a+2)(a+1 / 2) b$ we always get the ideal $\left(\lambda^{2}\right)$ which means that $T_{\lambda}^{(i)}$ cannot be singular.

\section{Convergent sextuples}

In this section we consider a special configuration of points.

Definition 4. Let $\mathcal{E}=\left(E_{0}, \ldots, E_{5}\right)$ be six points in $\mathbb{P}^{3}$ satisfying the following properties.

- All the points are distinct and all are different from the origin $O$;

- The three lines $E_{0}+E_{1}, E_{2}+E_{3}, E_{4}+E_{5}$ meet in a common, new point $A$;

- There are no other collinearities among the points;

- The six points are not contained in a plane.

The sextuple $\mathcal{E}$ will be called a convergent sextuple.

A simple example of a convergent sextuple is the following: 


$$
((1,0,0,1),(2,0,0,1),(0,1,0,1),(0,2,0,1),(0,0,1,1),(0,0,2,1))
$$

Convergent sextuples fulfil the following property.

Proposition 5. If $\mathcal{E}$ and $\mathcal{F}=\left(F_{0}, \ldots, F_{5}\right)$ are two convergent sextuples, then there exists precisely one projectivity which sends $E_{i}$ to $F_{i}, i=0, \ldots, 5$.

Proof. We call $R_{1}$ the intersection point of the lines $E_{0}+E_{3}$ with $E_{1}+E_{2}, R_{2}$ the intersection point of the lines $E_{0}+E_{5}$ with $E_{1}+E_{4}$, and $R_{3}$ the intersection point of $E_{2}+E_{5}$ with $E_{3}+E_{4}$. Then it is possible to verify that the lines $R_{1}+E_{4}$, $R_{2}+E_{2}$ and $R_{3}+E_{0}$ meet in a common point $A_{1}$. Let $A$ be the intersection point of the lines $E_{0}+E_{1}, E_{2}+E_{3}, E_{4}+E_{5}$ as in Definition 4. Then the five points $A, E_{0}, E_{2}, E_{4}, A_{1}$ are in generic position. Let now $S_{1}=\left(F_{0}+F_{3}\right) \cap\left(F_{1}+F_{2}\right)$, $S_{2}=\left(F_{0}+F_{5}\right) \cap\left(F_{1}+F_{4}\right)$ and $S_{3}=\left(F_{2}+F_{5}\right) \cap\left(F_{3}+F_{4}\right)$. Then, as above, the lines $S_{1}+F_{4}, S_{2}+F_{2}$ and $S_{3}+F_{0}$ meet in a common point $B_{1}$. Let $B$ the intersection point of $F_{0}+F_{1}, F_{2}+F_{3}, F_{4}+F_{5}$. Also now the points $B$, $F_{0}, F_{2}, F_{4}, B_{1}$ are in generic position, then, from the fundamental theorem of projectivities, there exists exactly one proiectively which sends $A$ to $B, E_{0}$ to $F_{0}, E_{2}$ to $F_{2}, E_{3}$ to $F_{3}$ and $A_{1}$ to $B_{1}$. It is easy to see that, consequently, $E_{1}, E_{3}, E_{5}$ are also sent to, respectively, $F_{1}, F_{3}, F_{5}$.

Remark 6. It is worthy of note a nice geometric property of a convergent sextuple: if we compute in the same way the point $A_{2}$ as the intersection of the three lines $R_{1}+E_{5}, R_{2}+E_{3}$ and $R_{3}+E_{1}$, then the points $A, A_{1}, A_{2}$ are collinear.

In every quartic surface $Q$ defined by (6) we can find convergent sextuples $\mathcal{E}$ with the further condition that the six lines $\left(E_{0}+E_{1}, E_{2}+E_{3}, E_{4}+E_{5}, E_{0}+\right.$ $\left.E_{2}, E_{3}+E_{5}, E_{1}+E_{4}\right)$ are contained in the surface. To see this, we can proceed as follows. Take three points $P_{i}, P_{j}, P_{k}$ among the 12 points of intersection of $F_{3}=0$ and $F_{4}=0$, with the following properties:

- $P_{i}, P_{j}, P_{k}$ are not collinear (i.e. $(i, j, k)$ is not in the list (3));

- for each of the three couples $\left(P_{i}, P_{j}\right),\left(P_{i}, P_{k}\right),\left(P_{j}, P_{k}\right)$ we can find a point $P_{i j}, P_{i k}, P_{j k}$ such that the triplets $\left(P_{i}, P_{j}, P_{i j}\right),\left(P_{i}, P_{k}, P_{i k}\right),\left(P_{j}, P_{k}, P_{j k}\right)$ are in (3).

The plane $O+P_{i}+P_{j}$ intersects $Q$ along the three lines $O+P_{i}, O+P_{j}, O+P_{i j}$ and a further line $\ell_{i j}$ which does not pass through $O$. Similarly, we find a line 
$\ell_{i k}$ and a line $\ell_{j k}$ of $Q$. Then a convergent sextuple is given by the points:

$$
\begin{aligned}
& E_{0}=\left(O+P_{i}\right) \cap \ell_{i j}, E_{1}=\left(O+P_{i}\right) \cap \ell_{i k}, \\
& E_{2}=\left(O+P_{j}\right) \cap \ell_{i j}, E_{3}=\left(O+P_{j}\right) \cap \ell_{j k}, \\
& E_{4}=\left(O+P_{k}\right) \cap \ell_{i k}, E_{5}=\left(O+P_{k}\right) \cap \ell_{j k} .
\end{aligned}
$$

A convergent sextuple of this form is called a standard convergent sextuple. For example, we can choose for $i, j, k$, the indices $0,1,3$ (in this order). In this case the points $E_{0}, \ldots, E_{5}$ are:

$$
\begin{aligned}
& (0,0,1,0),(0,0,1,4 a b),(1,0,1,0),(1,0,1,-(a+1) b), \\
& (0,1,1,2(2 a+1) b),(0,1,1,(2 a+1) b)
\end{aligned}
$$

The relevance of the standard convergent sextuples is given by the following proposition, whose proof is an immediate consequence of Proposition 5 .

Proposition 7. Suppose we have two quartic surfaces $Q=Q(a, b)$ and $Q^{\prime}=$ $Q\left(a^{\prime}, b^{\prime}\right)$ of the family (6), for $a, a^{\prime}, b, b^{\prime} \in K$. Then the two quartic surfaces are projectively equivalent if and only if we can find a standard convergent sextuple $\mathcal{E}$ on $Q$ and a standard convergent sextuple $\mathcal{E}^{\prime}$ on $Q^{\prime}$ such that the projectivity which sends $\mathcal{E}$ into $\mathcal{E}^{\prime}$ (according to Proposition 5) sends $Q$ to $Q^{\prime}$.

Let us point out another consequence of Proposition 5 . Fix a convergent sextuple $\mathcal{E}_{0}$, as in (7), and take a basic convergent sextuple $\mathcal{E}$ (as in (9)) on the quartic surface $Q(a, b)$. Then the projectivity which sends $\mathcal{E}$ to $\mathcal{E}_{0}$ transforms the quartic monoid $Q$ into a quartic monoid passing through the convergent sextuple (7). In other words, we can assume that all the quartic monoids pass through the sextuple (7), and, moreover, that such a convergent sextuple is a standard one.

The relevant fact is that in this way the equation of the transformed quartic monoid has a new equation which does not contain the parameter $b$ (as soon as we assume it is not zero). Therefore, all quartic surfaces of (6) obtained by different values of $b$ are projectively equivalent.

Here is the family of polynomials giving the quartic monoids for which (7) is a basic convergent sextuple: 


$$
\begin{aligned}
Q(a)= & 2 a x^{3} y+4 a x^{2} y^{2}+2 a x y^{3}-(2 a-1)(a-2) x^{3} z+ \\
& -\left(5 a^{2}-17 a+5\right) x^{2} y z-3\left(a^{2}-4 a+1\right) x y^{2} z+a y^{3} z+ \\
& -3(2 a-1)(a-2) x^{2} z^{2}-\left(7 a^{2}-19 a+7\right) x y z^{2}+2 a y^{2} z^{2}+ \\
& -2(2 a-1)(a-2) x z^{3}+a y z^{3}-2 a t x^{2} y-2 a t x y^{2}+2(2 a-1)(a-2) t x^{2} z+ \\
& 4\left(a^{2}-3 a+1\right) t x y z-2 a t y^{2} z+2(2 a-1)(a-2) t x z^{2}-2 a t y z^{2}
\end{aligned}
$$

The polynomial $Q(a)$ is obtained from the basic convergent sextuple of (6) constructed from the triplet $(11,10,9)$, since this choice allows us to obtain a simpler equation for $Q(a)$. Clearly, we assume $a \notin D=\{-1,0,1 / 2,1,2\}$ so that the surface $Q(a)$ is smooth outside the origin and its 31 lines do not degenerate. We have therefore the following:

Theorem 8. Let $K$ be a field of characteristic zero and let $a \notin\{-1,0,1 / 2,1,2\}$. Then all quartic monoid surfaces of $\mathbb{P}_{K}^{3}$ with no other singularities outside of the the triple point and with the maximum number of lines are projectively equivalent to a surface of equation (10).

Finally, the points $\bar{P}_{0}, \ldots, \bar{P}_{11}$ of intersection of the 12 lines of $Q(a)$ passing through the origin with the plane $t=0$ have now the following coordinates:

$$
\begin{aligned}
& (0,1,-1,0),(1,-1,0,0),(1,0,-1,0),(1,2 a-1,-a, 0),(1, a-2,1,0), \\
& (a,-2 a+1,-1,0),(1, a-2,-a, 0),(a,-a+2,-1,0),(a,-2 a+1, a, 0), \\
& (0,0,1,0),(0,1,0,0),(1,0,0,0)
\end{aligned}
$$

They still respect the collinearities given by (3).

\section{Stabilisers}

Starting from relations (3), it is possible to construct all the triplets of points $(i, j, k)$ with the property introduced in the previous section, i.e. such that the sextuple $\mathcal{E}$ given by (8) is standard and convergent in the sense of Definition 4 . Note that the number of the possible triplets is 720 (indeed, any set of points $\{i, j, k\}$ gives six standard convergent sextuples, one for each permutation of the indices $i, j, k)$.

Let $\mathcal{M} \in \mathrm{PGL}(4, K)$. We consider the natural action of the matrix $\mathcal{M}$ on $\mathbb{P}_{K}^{3}$ : given a point $R$ in $\mathbb{P}_{K}^{3}$ of coordinates $\left(u_{0}, u_{1}, u_{2}, u_{3}\right)$, we send it to the point $\mathcal{M} \cdot R$, whose coordinates are (the transpose of) $\mathcal{M} \cdot{ }^{t}\left(u_{0}, u_{1}, u_{2}, u_{3}\right)$. Hence PGL $(4, K)$ also acts on homogeneous polynomials in a natural way: if $F(x, y, z, t)$ is a homogeneous polynomial, then $\mathcal{M} \cdot F$ is the polynomial $F\left(\mathcal{M}^{-1} \cdot{ }^{t}(x, y, z, t)\right)$. According to this definition, if a point $R \in \mathbb{P}^{3}$ is a zero 
of $F$, then the point $\mathcal{M} \cdot R$ is a zero of $\mathcal{M} \cdot F$.

Let us consider the following problem: given a quartic monoid $Q(a)(a \in$ $K \backslash D)$, compute its stabiliser w.r.t. the action of PGL $(4, K)$, i.e. the group (in the following, by $\mathcal{M} \cdot Q(a)=Q(a)$ we mean that the surfaces defined by the equations $Q(a)=0$ and $\mathcal{M} \cdot Q(a)=0$, respectively, coincide)

$$
G_{a}=\operatorname{Stab}_{\mathrm{PGL}(4, K)} Q(a)=\{\mathcal{M} \in \mathrm{PGL}(4, K) \mid \mathcal{M} \cdot Q(a)=Q(a)\}
$$

The quartic monoid surface $Q(a)$ has the convergent sextuple $\mathcal{E}_{0}$ as in (7) among its standard convergent sextuples. If $\mathcal{E}$ is another standard convergent sextuple of $Q(a)$, let $\mathcal{M}$ be the unique (up to a multiplicative constant) matrix such that $\mathcal{M} \cdot \mathcal{E}_{0}=\mathcal{E}$ (see Proposition 5). If we compute $Q_{\mathcal{M}}=\mathcal{M} \cdot Q(a)$, we have to select those matrices $\mathcal{M}$ such that $Q_{\mathcal{M}}$ and $Q(a)$ define the same surface (moreover, we can also check if there are some specific values of $a$ such that for that specific value $Q_{\mathcal{M}}$ and $Q(a)$ coincide). Of course, this construction has to be repeated for the 720 standard convergent sextuples $\mathcal{E}$ of $Q(a)$. The computations are not too hard; the obvious way to see when $Q_{\mathcal{M}}$ and $Q(a)$ define the same surface, is to construct the $2 \times 35$ matrix $W=\left(w_{r s}\right)$ whose rows are, respectively, the coefficients of $Q_{\mathcal{M}}$ and of $Q(a)$ w.r.t. the 35 monomials of degree 4 in the variables $x, y, z, t$. The two surfaces coincide if and only if the rank of this matrix is one. In principle, the number of minors (595) might seem problematic, but the computation can greatly be reduced by the following observations: (i) in the matrix $W$ we can erase all the columns in which both entries are 0; (ii) if in the matrix $W$ the element $w_{1 s}$ is not zero but the element $W_{2 s}$ is zero, then the two surfaces cannot coincide and no other computations are needed; (iii) if $w_{1 s}=0$, then among the equations which test the coincidence of the two surfaces, we have to add $w_{2 s}=0$.

The following result holds true.

Theorem 9. If $a \in K \backslash\{-1,0,1 / 2,1,2\}$ is not a root of $x^{2}-x+1=0$, then the stabiliser $G_{a}$ of $Q(a)$ is isomorphic to the group $S_{3}$, and is generated by the following two matrices:

$$
\left(\begin{array}{rrrr}
2 & 2 & 2 & 0 \\
0 & -2 & 0 & 0 \\
-2 & 0 & 0 & 0 \\
0 & 1 & 3 & -2
\end{array}\right), \quad\left(\begin{array}{rrrr}
2 & 2 & 2 & 0 \\
0 & -2 & 0 & 0 \\
0 & 0 & -2 & 0 \\
0 & -2 & -3 & 2
\end{array}\right)
$$

If $a=\varepsilon$ is a solution of the equation $x^{2}-x+1=0$, then the equation of the quartic monoid surface $Q(\varepsilon)$ becomes $Q^{\prime}=0$ where: 


$$
\begin{aligned}
Q^{\prime}= & x^{3} y+2 x^{2} y^{2}+x y^{3}+3 / 2 x^{3} z+6 x^{2} y z+9 / 2 x y^{2} z+1 / 2 y^{3} z+9 / 2 x^{2} z^{2} \\
& +6 x y z^{2}+y^{2} z^{2}+3 x z^{3}+1 / 2 y z^{3}-x^{2} y t-x y^{2} t-3 x^{2} z t-4 x y z t \\
& -y^{2} z t-3 x z^{2} t-y z^{2} t
\end{aligned}
$$

In this case, its stabiliser $G_{\epsilon}$ has order 18, is isomorphic to $S_{3} \times C_{3}$ and is generated by the following two matrices:

$$
\left(\begin{array}{rrrr}
2 & 2 & 2 & 0 \\
0 & -2 & 0 & 0 \\
0 & 0 & -2 & 0 \\
0 & -2 & -3 & 2
\end{array}\right), \quad\left(\begin{array}{rccc}
0 & 2 \epsilon-2 & 2 \epsilon-4 & 0 \\
0 & -4 \epsilon+2 & 0 & 0 \\
2 \epsilon-4 & 2 \epsilon-2 & 0 & 0 \\
-3 & -3 \epsilon & -3 \epsilon & 4 \epsilon-2
\end{array}\right)
$$

We finally complete Theorem 8 answering the following question: how many vales of $a$ in 10 give the same quartic monoid up to a projectivity?

We can answer the question by mean of computations quite similar to the previous ones. Let $\mathcal{E}_{0}$ be the convergent sextuples given by (7) (remember that $\mathcal{E}_{0}$ is a standard convergent sextuple for all the surfaces of the family (10)). If $Q(a)$ and $Q(b)$ are two surfaces of the family 10 which are projectively equivalent, then there exists a standard convergent sextuple $\mathcal{E}$ of $Q(a)$ such that $\mathcal{M} \cdot Q(a)$ and $Q(b)$ coincide, where $\mathcal{M}$ is the $4 \times 4$ matrix which sends $\mathcal{E}$ into $\mathcal{E}_{0}$.

By repeating the above computation for all the 720 standard convergent sextuples and by selecting the cases leading to a positive answer to the question, we get the following result.

Theorem 10. Let $a, b \in K \backslash\{-1,0,1 / 2,1,2\}$ be two elements and consider the two quartic surfaces $Q(a)$ and $Q(b)$ from the family 10$)$. Then $Q(a)$ and $Q(b)$ are projectively equivalent if and only if a has one of the following values:

$$
b, \quad \frac{1}{b}, \quad \frac{1}{1-b}, \quad \frac{b}{b-1}, \quad 1-b, \frac{b-1}{b}
$$

We conclude by noticing how conditions 12 show that the action of PGL $(4, K)$ on quartic monoid surfaces of $\mathbb{P}_{K}^{3}$ with maximum number of lines is quite similar to the action of PGL $(3, K)$ on cubic curves of $\mathbb{P}_{K}^{3}$ (see [8], Lecture 10). In particular, two quartic monoid surfaces $Q(a)$ and $Q(b)$ are projectively equivalent if and only if $a$ and $b$ have the same $j$-invariant.

Remark 11. Among the lines of $Q(a)$, the lines $O+\bar{P}_{2}, O+\bar{P}_{9}$ and $O+\bar{P}_{11}$ can be distinguished from the others: they are the only three lines through the 
singular point of $Q(a)$ which intersect only 4 other lines of $Q(a)$ (see (3) or figure 11). The group $G_{a}$ corresponds to the permutations of these three lines. Analogously, the group $G_{\epsilon}$ corresponds to the permutations of the three lines above and the cyclic permutation of the three lines $\bar{P}_{1}+\bar{P}_{3}+\bar{P}_{7}, \bar{P}_{0}+\bar{P}_{5}+\bar{P}_{6}$, $\bar{P}_{4}+\bar{P}_{8}+\bar{P}_{10}$.

\section{References}

[1] J.

Abbott,

A. M. Bigatti, and L. Robbiano. CoCoA: a system for doing Computations in Commutative Algebra. Available at http://cocoa.dima.unige.it.

[2] M.C. Beltrametti, E. Carletti, D. Gallarati, and D. Monti Bragadin. Lectures on Curves, Surfaces and Projective Varieties - A Classical View of Algebraic Geometry. European Mathematical Society, Textbooks in Mathematics, 9, Zurich, 2009. Translated by F. Sullivan.

[3] M.C. Beltrametti, A. Logar, and M.L. Torrente. Complete intersection of cubic and quartic curves and a classification of the configurations of lines of quartic monoid surfaces, (2019). In preparation.

[4] F. Conforto. Le superficie razionali. Zanichelli, 1939.

[5] A.I. Degtyarev. Classification of quartic surfaces that have a nonsimple singular point. Math. USSR-Izv, 35(3):607-627, 1990.

[6] C.M. Essop. Quartic surfaces with singular points. Cambridge Univ., Press, 1916.

[7] V. Gonzalez-Alonso and S. Rams. Counting lines on quartic surfaces. Taiwanese J. Math, 20(4):769-785, 2016.

[8] J. Harris. Algebraic Geometry, a first course. Springer-Verlag, 1992.

[9] P.H. Johansen, M. Loberg, and R. Piene. Monoid hypersurfaces. Springer (ISBN 978-3-540-72184-0/hbk), pages 55-77, 2008.

[10] I. Polo-Blanco, M. van der Put, and J. Top. Ruled quartic surfaces, models and classification. Geom. Dedicata, 150:151-180, 2011.

[11] K. Rohn. Ueber die flächen vierter Ordnung mit dreifachem Punkte. Math. Ann., 24:55-151, 1884.

[12] W.A. Stein et al. Sage Mathematics Software (Version 6.7). The Sage Development Team, 2015. http://www. sagemath.org.

[13] T. Takahashi, K. Watanabe, and T. Higuchi. On the classification of quartic surfaces with triple point. I. Sci. Rep. Yokohama Nat. Univ., 29:47-70, 1982.

[14] T. Takahashi, K. Watanabe, and T. Higuchi. On the classification of quartic surfaces with triple point. II. Sci. Rep. Yokohama Nat. Univ., 29:71-94, 1982. 